February 25, 2005 13:30 WSPC/INSTRUCTION FILE mccollum04

Journal of Integrative Neuroscience

(C) Imperial College Press

\title{
DYNAMICS OF EVERYDAY LIFE: RIGOROUS MODULAR MODELING IN NEUROBIOLOGY BASED ON BLOCH'S DYNAMICAL THEOREM
}

\author{
GIN MCCOLLUM* \\ Neuro-Otology Research, Legacy Research Center \\ $1225 \mathrm{NE} 2^{\text {nd }}$ Avenue,Portland, Oregon, 97232 USA mccollum@ohsu.edu \\ PATRICK D. ROBERTS \\ Neurological Sciences Institute, Oregon Health and Sciences University \\ 505 NW 185 ${ }^{\text {th }}$ Avenue, Beaverton, Oregon, 97006 USA, robertpa@ohsu.edu \\ http://www.ohsu.edu/nsi/faculty/robertpa/
}

Received 14 April 2003

Accepted 5 August 2003

\begin{abstract}
Natural, everyday sensorimotor behaviors, such as rising from sitting, typically have an intrinsic organization of several levels of analysis. Taking this intrinsic organization as key to understanding neural dynamics is neither a top-down nor a bottom-up approach, but rather a meshing of multiple centers and levels of analysis. Motor control requires body dynamics that are consistent with physical dynamics, besides the more microscopic levels of neural dynamics. The dynamics of separate movements have been investigated as if the ends can be capped off, separated from the rest of the individual's life. Is this dynamically correct? Even chaotic behavior is deterministic. However, the mathematics of nonlinear oscillations is not all of dynamics. This paper relates Bloch's dynamical theorem to the modular, conditional approach to sensorimotor and other neural functioning.

Bloch's dynamical theorem lays a foundation for the piecewise study of structurally accurate dynamics in theoretical neurobiology. Piecewise studies can be used as a modeling option complementary to the methods of nonlinear oscillator dynamics. By applying Bloch's theorem, dynamics of movements analyzed piecewise can be extended into a smooth flow on any manifold, either as a whole or conditionally. Conditional dynamics makes dynamical modeling options explicit, often depending on what variables the organism can control, and allows one to take different modeling options at different junctures in analyzing the same phenomenon. For example, this approach allows the study of complex motor control problems to be reduced to modular constructions using singularities and flow lines. Dynamical contingencies are expressed using the mathematics of ordered structures. This paper presents Bloch's dynamical theorem and its relevance to model construction in theoretical neurobiology. Specific examples, integrated into physiological and behavioral context, are cited from the literature.
\end{abstract}

Journal of Integrative Neuroscience, Vol. 3, No. 4 (2004) 397-413

Keywords: Dynamical systems; dynamics; mathematics; conditional.

${ }^{*}$ Corresponding author. 


\section{Introduction}

The success of dynamical systems mathematics in yielding insight into cardiology, bursting in excitable cells, and rhythmogenesis in neuronal networks has raised hopes of the broad applicability of similar mathematics in neurobiology. In particular, dynamics has appeared to be a more biology-friendly approach, more conducive to insight into such quintessentially biological phenomena as self-organization [45]. Bloch's dynamical theorem on the extension of stable flows from restricted regions [3] provides a powerful tool for structurally accurate analysis in neural systems. This paper begins with a brief review of the applications of dynamical systems mathematics in physiology as a context for presenting Bloch's dynamical theorem and its implications in neurobiology.

Rhythmic movements can be conceptualized as having settled into an attractor in a dynamical space. For example, consider the waggling motion of a forefinger rigidly oscillated about the metacarpo-phalangeal joint in the plane of the palm. When both forefingers are waggled at the same time, they may have a variety of phase and frequency relationships. Two phase relationships are preferred in the 1-2 $\mathrm{Hz}$ frequency range, 0 and 180 degrees out of phase (Fig. 1A), so that they can be considered as a pair of attractors or potential wells (Fig. 1B) [43]. At higher frequencies, 2.5-3 Hz, the 0-degree, in-phase movement is preferred (Fig. 1C), so that the 0 -degree potential well is much deeper or the 180-degree attractor basin spills into that of the 0 -degree phase relationship.

The patterns characterizing phase relationships are more complicated in locomotor rhythms of quadrupeds; the patterns are called gaits, and depend upon frequency as do the finger-waggling phase relation preferences. Mathematical symmetry considerations introduce both specificity and mathematical depth to the modeling of locomotor gaits $[44,7,8]$. Symmetry methods have been used elegantly to determine the minimal neural network with the appropriate symmetry to produce gait patterns for animals with paired legs [13].

Oscillatory patterns are produced by a range of neural and other excitable systems, leading to a rich modeling literature using methods of nonlinear oscillator dynamics. For example, oscillatory patterns in cardiac cells and tissue include phase locking and period doubling [17] and low-dimensional chaos [6]. These cardiac studies benefit from the extensive data sets available on the steadily-beating heart. The sensitivity of oscillatory behaviors and nonlinear systems methods suits them to the study of underlying biochemical mechanisms, such as ion channels $[5,15]$ and calcium handling [20, 21, 22].

Detailed phase plane models of excitability are characterized by a double attractor, in which the flow pattern is subject to large excursions from a steady state in response to small perturbations (Fig. 2) [46, 11]. Dynamical studies have led to a tentative taxonomy of bursting mechanisms in excitable systems affected by processes on different time scales $[39,9]$. These studies investigate the behavior of flows within attractor basins. 
A

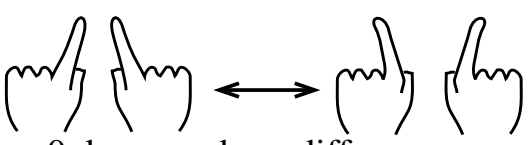

0 degrees phase difference

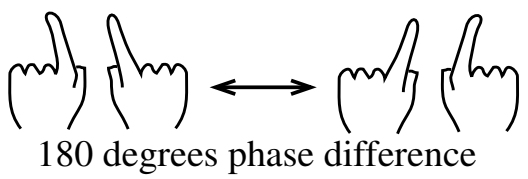

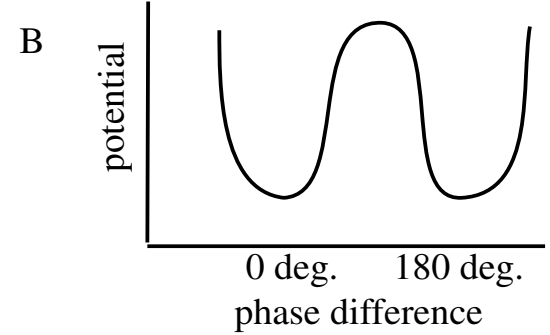

$\mathrm{C}$

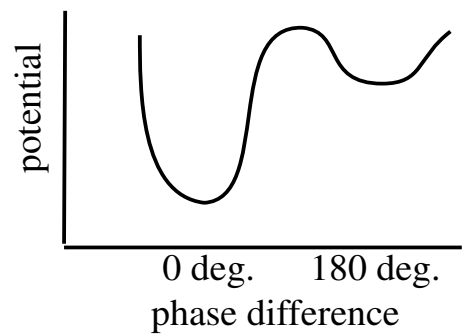

Fig. 1. Phase relations of finger-waggling. A. Explanations of phase conventions. B. Potential wells for low-frequency finger-waggling. Neither phase relation is significantly preferred over the other, although 0 and 180 degrees are preferred over other phase relations. C. Change in phasing preference at higher frequencies. The preference for $0 \mathrm{deg}$. phase difference is represented by a deeper potential well.

The mathematics of dynamics offers a flexible tool for modeling with structural accuracy both the attractors/semistable states and the flows between. Attractors in neurobiology occur at several levels of analysis, including the subcellular, cellular, neuronal population, and organism levels. On all these levels of analysis, the broader study of dynamical systems investigates the overall nature of flows within which attractor basins occur. For example, in Figure 3, the attractor basin of erect stance is included in a flow in which some trajectories reach standing and some do not. (It is the attractor basin and not the point sink that is important here, because there are more detailed dynamics within the basin, as discussed further in section IV.) The dynamical flow displays a range of alternative trajectories reaching the standing attractor from the sitting position [40]. Sitting and standing are examples of the many sensorimotor states an animal remains in temporarily in the course of its behavior [19, 38, 37, 14, 24]. Although numerical aspects such as forces and lengths may vary for different examples of a behavior such as standing up from sitting, it is structural accuracy that is essential to understanding the behavior. That is, the structure of the mathematics must reflect the organization of the physiological system [26, 27, 28, 30].

Bloch's theorem, presented in the next section (2), addresses the manifolds to which flows may be extended, in terms of basic concepts of dynamics. Dynamical flows include not only attractors, but also repellers and saddles (Fig. 4). Attractors, repellers, and saddles each maintain invariant a particular set of points (conventionally denoted $\Lambda$ ) under the action of the flow. Besides the idea of invariance, which is 


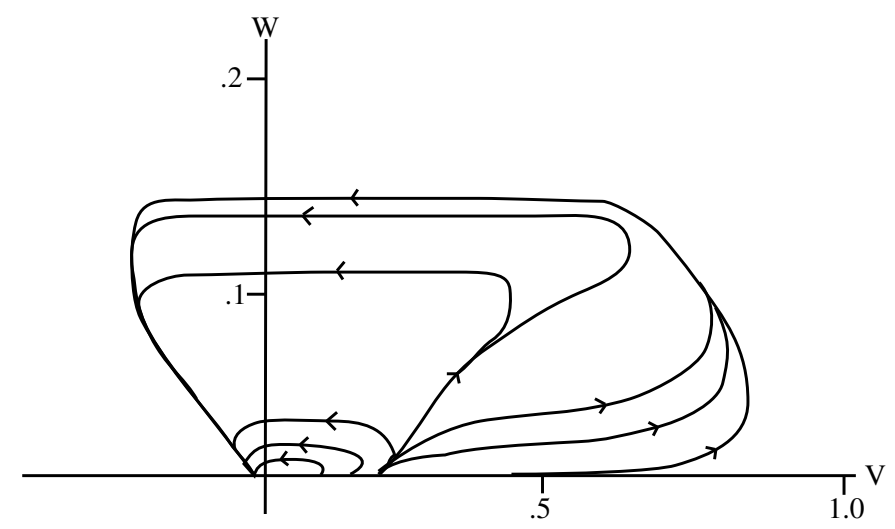

Fig. 2. Phase plane diagram showing the qualitative current-voltage cycling behavior typical of neurons in the FitzHugh-Nagumo model [11]. W and V are generalized coordinates representing current and voltage, respectively, in the linearized case. With low voltage perturbations, current perturbations increase modestly with voltage. At threshold (around.25), current perturbations suddenly increase nonlinearly. (Modified from [39])

a more general one in mathematics, the mathematics of dynamics considers metric properties of the flow. Under the flow, an arbitrarily small neighborhood within a nonwandering set (conventionally denoted $\Omega$ ) returns and intersects itself repeatedly and indefinitely. Nonwandering sets are the important ones, because other points either wander off to infinity (in a non-compact manifold) or end up in an attractor (especially within a compact manifold). Within an attractor basin, the flow is stable in the sense that it flows only inward, whereas both repellers and saddles are unstable.

Following the presentation of Bloch's theorem, section 3 presents further basic theorems and a discussion of extension in neurobiology modeling. The final section (4) presents options for using dynamics in neurobiology modeling.

\section{Stable Flows Extended from an Isolating Block}

Mathematics is based rigorously on definitions and axioms which may not apply exactly in empirical sciences such as physics and neurobiology. For example, since organisms are composed of molecules and smaller particles, it is not clear that continuity holds with microscopic exactitude. Therefore, continuity (and synonymously, smoothness) and other empirically questionable concepts will not be addressed here. "Compact" will be abbreviated, for biological purposes, to "finite". On the other hand, the mathematical constraint of structural stability is a useful one in identifying mathematics appropriate for modeling empirical systems, especially biological ones: a structurally stable flow is one which maintains its set of attractors, repellers, and saddles and the flows between them under small perturbations. For example, a dynamical model of the sit-to-stand movement should be approximately the same with small variations in the exact position of the center of mass of the body segments. 


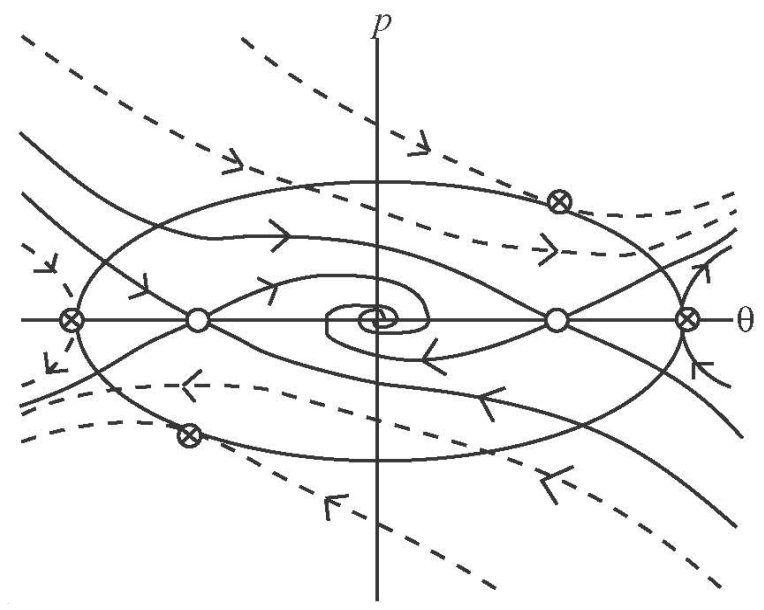

Fig. 3. Phase plane diagram showing rising from sitting. The generalized angular variable, $\theta$, is defined such that sitting is at the left of the diagram and standing is at the origin. Some trajectories lead into the standing attractor basin. On the vertical axis is the generalized angular momentum, $\mathrm{p}$, associated with. Adequate momentum is required to rise from sitting, as shown by trajectories between the solid branches departing from the upper left-hand side of the diagram. Dashed trajectories either return to a sitting position or bypass standing. The attractor and two saddle points (empty circles) are surrounded by an oval boundary, which can be used as the boundary of an isolating block (see text for description). The set of isolated tangent points of the isolating block are marked with circled x's.

It is also useful in biology to study an activity or phenomenon, such as erect stance, in isolation from what went before or will come after. This ability is conferred mathematically by an isolating block: a finite, bounded region $\mathrm{U}$ of the same dimensionality as the manifold $\mathrm{M}$ in which it is embedded, with a flow $\varphi$ on $\mathrm{U}$ such that no flow lines within $U$ are tangent at the boundary. Thus, the repeller and saddle in Figure 4 are drawn in isolating blocks, as is the region within the oval boundary in Figure 3. However, tangencies with one or both ends within the region disqualify the region as an isolating block (Fig. 5). The "standing" attractor (Fig. 3) can be enclosed in an isolating block, as a separable phenomenon, to be followed by a variety of alternative behaviors, such as sitting and walking.

Bloch's theorem can be summarized as: Given a flow on an isolating block, the flow can be extended to the rest of any manifold. Because, according to the first corollary [3], this is true of any finite number of isolating blocks, this means that any combination of attractors, repellers, and saddles can be specified separately and linked together.

With technicalities included, the theorem is:

Bloch's Theorem: Let $\mathrm{M}^{n}$ be a smooth closed manifold and let $\left(\Lambda, \varphi, \mathrm{U}^{n}\right)$ be a triple where $\varphi$ is a $\mathrm{C}^{r}$ flow, $\mathrm{r} \geq 1$, and $\mathrm{U} \subset \mathrm{M}$ is an isolating block. Then there exists an embedding of the flow $\varphi$ into a smooth Axiom A flow on $\mathrm{M}^{n}$. That is, there exists a smooth flow $\varphi^{\prime}$ on $\mathrm{M}^{n}$ such that:

(1) $\varphi^{\prime}$ is Axiom A. 

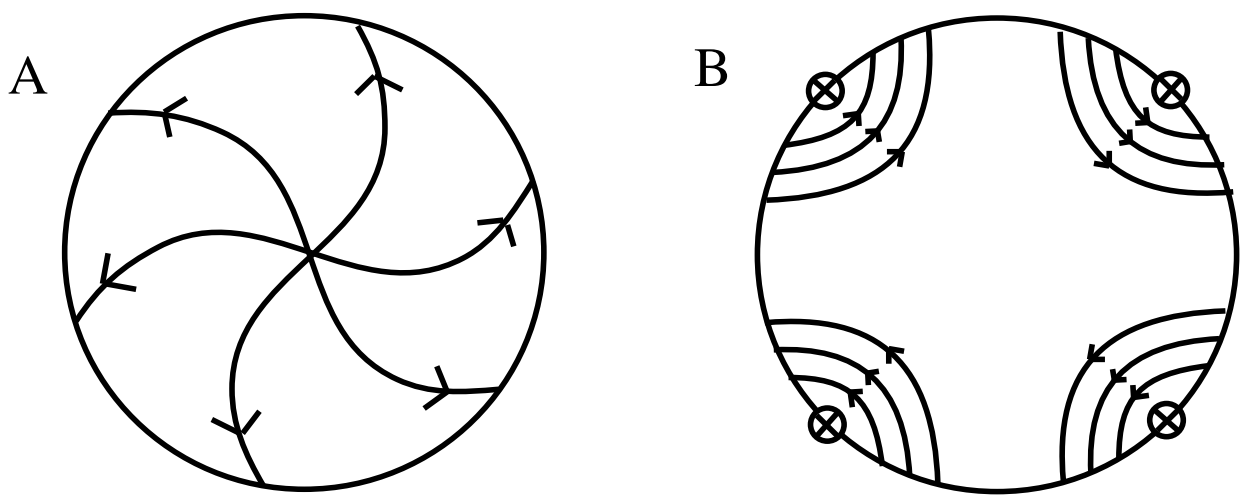

Fig. 4. Isolating blocks. A. Repeller. B. Saddle. At tangent points of the flow to the boundary, marked with circled x's, the flow lines are entirely external to the isolating block.

(2) $\left.\varphi^{\prime}\right|_{U}=\varphi$.

(3) The nonwandering set $\Omega\left(\varphi^{\prime}\right) \cap \mathrm{U}=\Lambda$.

(4) The extended flow $\varphi^{\prime}$ is $\Omega$-stable for all $\mathrm{n}$.

(5) For $n=2,3$ and $r>1$, the extended flow $\varphi^{\prime}$ is structurally stable.

Explanations: The $\mathrm{n}$ in $\mathrm{M}^{n}$ and $\mathrm{U}^{n}$ is not used strictly as an exponent, but rather denotes the dimensionality of the manifold. "Axiom A" is an adjective specifying the relationship of the rest of the manifold to the nonwandering sets. $\Omega$-stability is a limited form of structural stability, with stability under a small range of perturbations limited to the nonwandering set $\Omega$, as opposed to the whole manifold.

The proof of the theorem in two dimensions involves constructing a collar around the boundary of the isolating block that makes the isolating block plus the collar look like a repeller from the point of view of the rest of the manifold. This makes the rest of the manifold into a manifold with boundary, where the flow is coming in transversely to the boundary. On any such manifold, one can put a Morse-Smale flow, that is, one for which

(1) the number of fixed points and periodic orbits is finite and each is hyperbolic (like an attractor, repeller, or saddle);

(2) all stable and unstable manifolds intersect transversally;

(3) the nonwandering set consists of fixed points and periodic orbits alone [16].

A Morse-Smale flow is structurally stable. The proof of the theorem in higher dimensions uses similar techniques, except that in higher dimensions there are more types of hyperbolic fixed points and other, much more complicated invariant sets $\Lambda$.

In two dimensions, it is sufficient to consider repellers, attractors, and saddles. If $\Lambda$ is an attractor or repeller, the boundary of $U$ can be chosen so that the flow is never tangent to it. If $\varphi$ is a repeller, the extension can be made by simply continuing the flow across the boundary. If $\varphi$ is an attractor, it can be camouflaged by one or more repellers, to look like a repeller from outside the collar (Fig. 6). If $\Lambda$ is a saddle, there will be tangencies to the boundary of the isolating block; the boundary must 


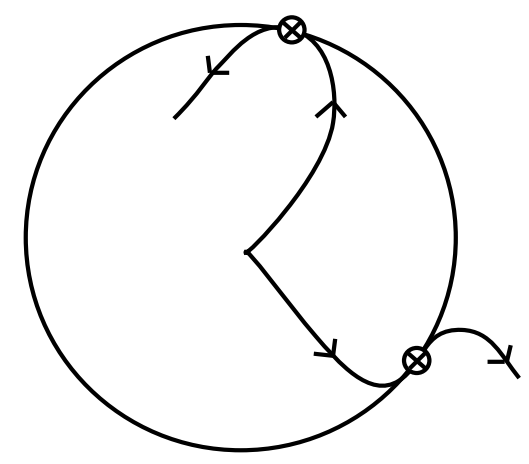

Fig. 5. Not an isolating block. Two tangent points of the flow to the boundary are shown. At one, the flow is internal to the boundary. At the other, the flow exits. Both of these flow lines are disallowed for an isolating block.

be chosen so that they are external, as in Fig. 4B. Still, to make absolutely certain that all the requirements are met, a saddle requires a more elaborate collar than an attractor (Fig. 7).

It is this type of extension that allows, for example, the sit-to-stand movement to be extended to a walk, handshake, or suitcase-picking-up movement. The denial of Bloch's theorem would say something like: every movement you make affects the rest of your life and the rest of the universe. That may be to some extent true, but Bloch's theorem allows a great deal of freedom, both of action and of neurobiology modeling.

\section{Extension in Neurobiology Modeling}

The freedom to extend the flow on an isolating block gives modellers the freedom to (1) characterize behavioral phases in isolation and then (2) join them with other behavioral phases. According to Bloch's theorem, once a modular movement is understood, if it can be enclosed in an isolating block, then it can be extended to other movements with a large amount of generality. Thus, it is not necessary to understand the dynamics of a system for all time, in order to characterize its local dynamics.

It is customary in physics to write dynamical equations that are doubly infinite in variables such as time, position, and momentum, that is, for which the variables extend to both negative and positive infinity. However, important topological theorems in dynamics require the state space to be a compact manifold, finite like a sphere or a torus, rather than an open space like $\mathfrak{R}^{n}$. These theorems, such as the Poincaré-Bendixson theorem and Peixoto's theorem, are powerful tools for characterizing local dynamics $[15,1]$. (See Appendix for the Poincaré-Bendixson theorem and Peixoto's theorem.)

Specifically, the Poincaré-Bendixson theorem limits a modeller's attention to a very restricted set of attractors, repellers, and saddles. For example, [40], in mod- 


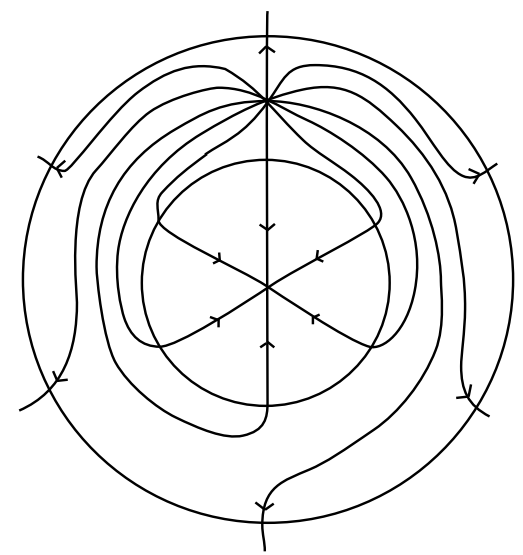

Fig. 6. Collar around an attractor within an isolating block. The inner circle is the boundary of the isolating block. The outer circle is the outer boundary of the collar.

eling the sit-to-stand movement, constrained the movement to a capped cylinder (topologically equivalent to a sphere), so that there were no limit tori, only limit points or limit cycles.

In the case of the sit-to-stand movement (Fig. 3), there are two saddles and one attractor. The attractor, at the position of erect stance, represents the postural adjustment mechanisms that maintain humans in the passively unstable standing position. Preliminary physical calculations attest to two saddles, one dividing the sit-to-stand movement from a failure in which the trajectory returns to the chair from want of momentum, and one on the far side of the erect stance attractor, dividing sit-to-stand movements from trajectories unsuccessfully approaching erect stance from the opposite direction. In order to know the combinations in which the attractor and saddles connect, another theorem is applied that assumes a compact manifold, Peixoto's theorem. According to Peixoto's theorem (Appendix), there are no connections between saddle points in a structurally stable flow.

Therefore, there are only three ways to connect the attractor and the two saddle points in a structurally stable flow: both saddles connecting to the attractor (Fig. 3), only the chair-side saddle connecting to the attractor (Fig. 8A), and only the far-side saddle connecting to the attractor (Fig. 8B). Mathematically, the first two are the only structurally stable dynamical flows leading from the chair to the erect stance attractor. It is to the advantage of neural control to choose a structurally stable flow, to avoid the vagaries attendant upon a movement that requires excessive precision. Therefore, these two flows specify the topology of the sit-to-stand movement.

The conclusion that there are only two dynamical flows for the sit-to-stand movement was made possible by limiting the range of momentum and considering a capped cylinder rather than a doubly-infinite cylinder. However, one may ask whether capping the cylinder - restricting attention to a finite area of the state space - leads to unacceptable dynamical consequences elsewhere, in the subject's 


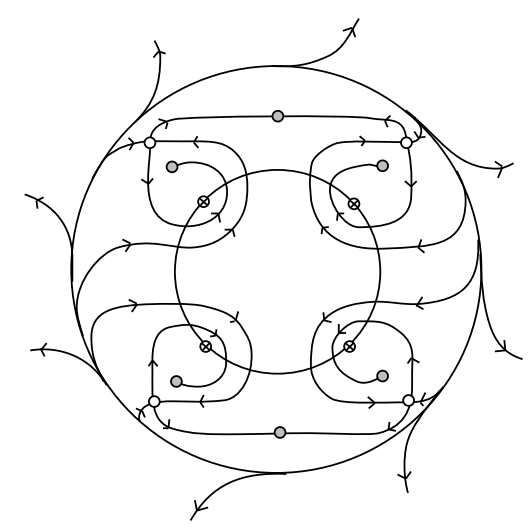

Fig. 7. Saddle with a collar, extended to a repelling flow.

past or future. Bloch's theorem answers that question: No. Bloch's theorem allows modellers to restrict attention to a finite area of a state space, in order to analyze it thoroughly, and then join the solution to solutions for other behaviors. The dynamics of multiphase movements are thus an interdependent combination of the dynamics of single movement phases, such as the sit-to-stand movement. This approach allows the study of complex motor control problems to be reduced to "tinker-toy" constructions using singularities and flow lines.

Just as for motor behaviors, modellers can restrict attention to subsets of a neural system, including a single neuron. The flexibility in modeling is reflected in the variability of combinations in which movements occur. For example, the most perfectly swung bat may not contact the ball; if it does make contact, the ball may yet go astray. Similarly, a neuron's activity may and may not arouse a response in the network to which it belongs, depending on the overall organization of the other neurons. For example, the dorsal gastric neuron in the stomatogastric ganglion of decapod crustaceans fires in a pattern to influence the gastric mill. It may fire alone. Alternatively, it may be an integral part of a network firing in concert to influence the gastric mill [10]. This observation may suggest alternative options for modeling: rather than attempting one grand model including both the neuronal and the network levels, a modeller may choose different methods to give insight into neuronal behavior and network behavior, then combine the results.

\section{Options for Neurobiology Modeling}

At least two complementary options have been used in structurally accurate neurobiology modeling: system as oscillator and system as navigating and interacting with dynamical conditions. For example, the locomotor step cycle of one leg has been considered as a limit cycle obeying oscillatory dynamics under particular constraints [34,4] (Fig. 9A). An alternative point of view has been to separate neural control from physical conditions. Without neural mechanisms of posture, standing 

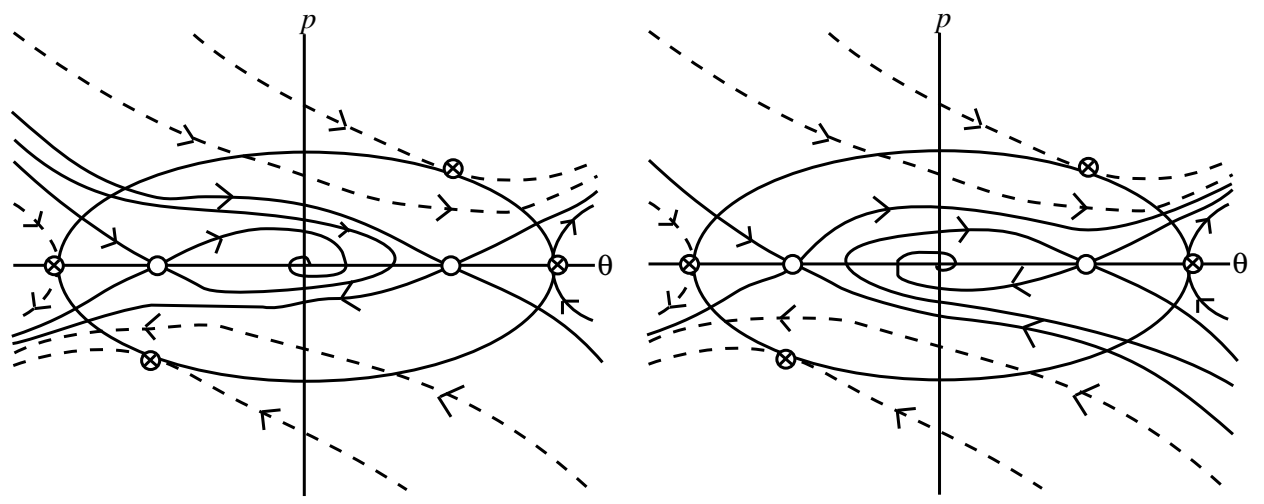

Fig. 8. Two more possibilities for connecting two saddles and an attractor in a structurally stable flow, in addition to Figure 3.

on a leg is unstable and can be represented as a repeller. A human locomoting, then, is navigating an alternation of two repellers (Fig. 9B). Achievement of independent locomotion has been modelled as the construction of a strategy for maintaining upright stance and forward motion under these physical conditions, using principles such as the conservation of potential plus kinetic energy and the conservation of angular momentum [32].

The two points of view are equally valid and yield different insights. They can be used at any level of analysis. Nervous systems are complex; we must expect multiple, complementary, mathematical points of view in theoretical neurobiology.(See [42], for another approach to discrete movements, not discussed here.)

\subsection{System as oscillator}

Contemplating celestial motions, Newton assumed that planetary orbits arise under the influence of an attracting force. A similar assumption is made about cyclic behavior in biology: that the behavior arises under the influence of an attractor, which is therefore the main subject of modeling. This approach has the advantage of using powerful mathematical methods that have become well established, largely because of the influence of Newtonian mechanics.

Some of the important models in cardiology and cell physiology that are based on this point of view and use the mathematics of nonlinear oscillators have been mentioned in the Introduction. These models directly represent the periodic behavior of hearts and cells. There are similarities and differences between these models and efforts to probe neural control of eye movements using an imposed sinusoid. In two standard paradigms, an animal attempts to visually fixate a target (humans, by instruction; non-humans, after training). The sinusoid is imposed either as a target motion or as a motion of the animal's head. In each case, the eye movement is analyzed as a function of the imposed sinusoid, using a Laplace transform, transfer function approach to constructing differential equations (for example: $[2,12]$ ). 
A

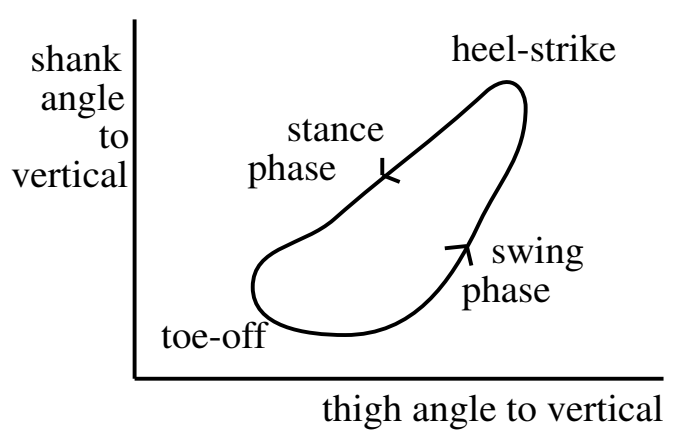

B

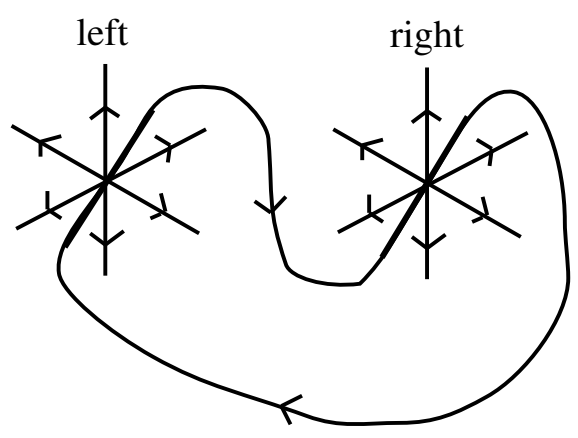

Fig. 9. Options for modeling locomotion, illustrated by schematic diagrams. A. As an oscillation. Although the cycle shown is parameterized by shank and thigh angles to vertical, variables used in experimental and theoretical studies may differ. B. As the navigation of physical dynamics. The unstable point of erect stance on one leg is represented by a simple repeller, one for each leg, right and left. The dashed line joins the two repellers through a space whose variables are unspecified in the diagram.

Like the cardiology and cell physiology models, these oculomotor control models use powerful and well- established mathematical methods. However, they are not direct models of the behavior of interest, but attempts to probe neural behavior not available for direct observation, by imposing sinusoids and other particular conditions. Complementary models will be required to give insight into the dynamics of the neurons and neuronal populations involved in oculomotor control and into the dynamics of non-oscillatory and voluntary eye movements.

\subsection{System as navigating and interacting with dynamical conditions}

Just as sinusoids can be imposed as conditions on eye movement control, so can non-sinusoids be imposed as conditions. Conditional dynamics makes dynamical modeling options explicit and allows one to take different options for different aspects of the model, depending, for example, on which variables the organism can control. In order to make dynamical modeling options explicit, conditions are expressed using algebraic relations: inclusion to express "governed by" and contiguity to express "lead to" [23, 24, 25, 29, 27, 28]. For example, rising from sit to stand changes the sensorimotor state of the organism, from a sitting to a standing state. By entering a subset of the sitting state (producing adequate forward momentum), a person can take advantage of the range of dynamic trajectories that lead to standing (Fig. 10). These trajectories are governed by the overall dynamics of the sit-to-stand movement, which includes both the sitting and standing positions.

Included in the standing attractor basin, at a more detailed level of modeling than Figure 3, is a system of postural adjustments depending on and regulated by motor, sensory, and environmental conditions. An example of a motor condition 
would be the position of the feet: with the feet side by side, a sideways perturbation could be countered by differential pressure on the two feet, whereas with one foot ahead of the other, the postural adjustment countering a similar perturbation would be a more essentially hip movement. An example of a sensory condition would be whether visual cues are available and reliable as orientation cues. An example of an environmental condition would be whether the support surface is slippery, like ice, or whether it affords friction so that shear forces can be relied on as reaction forces to postural adjustments, especially those involving hip movements. The postural adjustments in each of these conditions, such as those involving hip or ankle torque, can be analyzed piecewise, according to Bloch's theorem, and then extended to lead to other movements.

\subsection{Modeling when dynamics is unknown or complicated}

The dynamics of neuronal populations, circuits, and networks are often inadequately understood for direct modeling. However, a great deal may be known, piecewise, about various aspects of the organism, the behavior mediated, and individual neurons. Then conditional dynamics can be used to characterize the system as a whole, leading to implications about relationships between the parts.

A great deal is known, for example, about the stomatogastric ganglion of decapod crustaceans and the food-processing behaviors it mediates (for reviews, see [18]). The neuronal and food-processing behaviors have been combined in one model, using conditional dynamics [41].

By expressing dynamical contingencies using the mathematics of ordered structures, plus contiguity, conditional dynamics introduces mathematical structure for both characterizing and analyzing control structures. Control structures may switch controlled variables, movements, and sensory cues for perception and movement, depending on conditions such as those listed above for standing postural adjustments. A distributed control structure for such a conditional system of controls must be organized to produce the observed smoothness of control. For human postural adjustments, that level of smoothness is very high. Therefore, postural adjustment control structures have been modelled to satisfy constraints that ensure smooth and appropriate switching between different dynamical responses [33, 25, 27].

The control system of standing postural adjustments contain only some of the discrete states presented in the literature. Sleeping and waking are two distinct states we switch between. Prochazka $[38,37]$ has characterized discrete states of motor control and suggested that the switching between them may be controlled by a conditional logic [36]. reported Switching between sensorimotor states in fish was reported in [19]: a vestibularly-dominated state identified by tilting in vestibulardamaged fish and a visually-dominated state with no tilting, which occurred when the fish swam toward food. Neural switching of reference frames in the hippocampus

have recently been reported [14], contingent on the movement and training of the animal. Although these systems may not be tractable using nonlinear oscillator ap- 


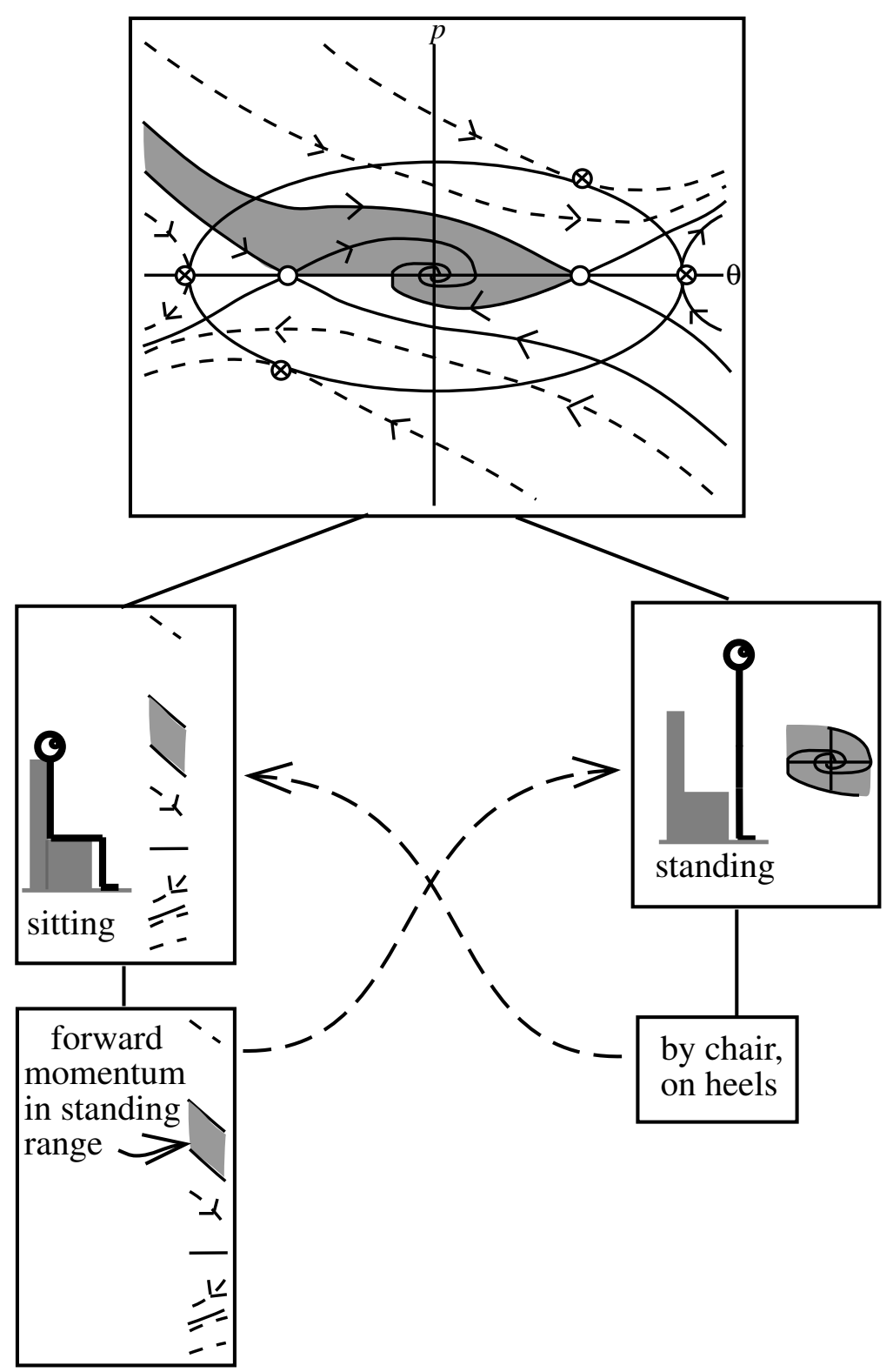

Fig. 10. Sit-to-stand movement as a transition between states, governed by dynamics. This diagram expresses the conditional dynamics of transitions between sitting and standing, and is to be read as a mathematical expression, like an equation. The conditional dynamics is expressed as relations between dynamical regions, each denoted within a box. The dynamical flow governing the sit-to-stand movement is shown at the top of the page, with the range of angular momenta leading to standing shaded. The phase plane includes both the standing state (the attractor in the middle) and sitting (the left-hand edge). Inclusions are denoted by solid lines, as is conventional in the mathematics of ordered structures, with the including set higher on the page. For example, included in the region labelled "sitting" is the subset of sitting that has angular momenta within the range leading to standing. This subset is called a "trigger region" because it leads to a state transition, between sitting and standing. Contiguity is denoted by dashed arrows, such as the one leading from the sitting trigger region to the standing state. An analogous trigger region will be revealed by dynamical analysis of the stand-to-sit movement; here, it is indicated by "by chair, on heels". Although the sit-to-stand phase plane includes the trigger region for the stand-to-sit movement, it does not represent the necessary dynamical flow. The two states, sitting and standing, plus the 
proaches to modeling, they may yet be open to piecewise modeling using conditional dynamics.

Neurobiology is a rich field. It is not to be expected that one mathematical approach will solve all problems. (And numerical computation alone certainly will not, even though it is currently popular.) Rather, we can aspire to create a collage of intersecting, structurally accurate mathematical characterizations, that shift to give complementary insights from different points of view. Bloch's theorem lays a foundation for piecewise analysis of dynamical behavior at any level of analysis and its extension to connect with interdependent behaviors.

\section{Appendix: Dynamical Theorems}

Poincaré-Bendixson Theorem: For vector fields on compact, orientable, twodimensional manifolds, limit sets must be limit cycles, limit points, limit tori, or combinations of points and connecting arcs. (A non-orientable manifold has a twist, like a Möbius strip or a Klein bottle. A sphere is orientable.)

Peixoto's Theorem: A smooth vector field on a two-dimensional compact manifold is structurally stable if and only if:

1. The limit sets consist only of fixed points and periodic orbits (limit cycles).

2. The number of fixed points and closed orbits is finite and each is elementary.

3. There are no connections between saddle points.

Furthermore, if the manifold is orientable, structurally stable flows are generic in the space of all two-dimensional flows.

\section{Acknowledgments}

We are indebted to Alan Garfinkel and Gerhard Magnus for discussions of the dynamics literature, and to Gerhard Magnus and William L. Bloch for critique of the manuscript. Early discussions with Jan Holly sharpened PDR's awareness of the need for a theorem like Bloch's. This work was supported in part by the National Institutes of Health under Grant No. R01-MH60364 to PDR.

\section{References}

[1] Abraham R, Marsden JE, Foundations of Mechanics, 2nd ed., Addison-Wesley Publishing Company, Reading, Massachusetts,1985.

[2] Bilotto G, Goldberg J, Peterson BW, Wilson VJ, Dynamic Properties of Vestibular Reflexes in the Decerebrate Cat Exp Brain Res 47: 343-52, 1982.

[3] Bloch WL, Extending Flows from Isolated Invariant Sets Erg Th Dyn Sys 15: 10311043, 1995.

[4] Borghese NA, Bianchi L, Lacquaniti F, Kinematic Determinants of Human Locomotion J. Physiol. 494: 863-879, 1996.

[5] Chay TR, Fan YS, Lee YS, Bursting, Spiking, Chaos, Fractals, and Universality in Biological Rhythms Int J Bifur Chaos 5: 595-635, 1995. 
[6] Chialvo DR, Gilmour RF Jr, Jalife J, Low Dimensional Chaos in Cardiac Tissue Nature 343: 653-657, 1990.

[7] Collins JJ, Stewart IN, Symmetry-breaking bifurcation: A possible mechanism for 2:1 frequency-locking in animal locomotion $J$ Math Biol 30: 827-838, 1992.

[8] Collins JJ, Stewart IN, Coupled nonlinear oscillators and the symmetries of animal gaits Nonlinear Sci 3: 349-392, 1993.

[9] Del Negro CA, Hsiao C-F, Chandler SH, Garfinkel A, Evidence for a Novel Bursting Mechanism in Rodent Trigeminal Neurons Biophys J 75: 174-182, 1998.

[10] Dickinson PS, Moulins M, Interactions and Combinations between Different Networkd in the Stomatogastric Nervous System, in Harris-Warrick RM, Marder E, Selverston AI, Moulins M(eds.) Dynamic Biological Networks: The Stomatogastric Nervous System The MIT Press, Cambridge, Massachusetts, 1992.

[11] FitzHugh R, Mathematical Models of Excitation and Propagation in Nerve, in Biol Eng, Schwan HP(ed.) McGraw-Hill, New York, 1969.

[12] Furman JM, Hain TC, Paige GD, Central Adaptation Models of the Vestibulo-Ocular and Optokinetic Systems Biol Cybern61: 255-264, 1989.

[13] Golubitsky M, Stewart I, Buono P-L, Collins JJ, A Modular Network for Legged Locomotion Physica D 115: 56-72, 1998.

[14] Gothard KM, Skaggs WE, McNaughton BL, Dynamics of Mismatch Correction in the Hippocampal Ensemble Code for Space: Interaction between Path Integration and Environmental Cues J Neurosci 16: 8027-8040, 1996.

[15] Guckenheimer J, Gueron S, Harris-Warrick RM, Mapping the Dynamics of a Bursting Neuron Phil Trans Royal Soc (London) B 341 :345-359, 1993.

[16] Guckenheimer J, Holmes P, Nonlinear Oscillations, Dynamical Systems, and Bifurcations of Vector Fields 3rd ed., Springer-Verlag, New York,. 1983

[17] Guevara MR, Glass L, Shrier A, Phase Locking, Period-Doubling Bifurcations, and Irregular Dynamics in Periodically Stimulated Cardiac Cells Science 214: 1350-1353, 1981.

[18] Harris-Warrick RM, Marder E, Selverston AI, Moulins M, Dynamic Biological Networks: The Stomatogastric Nervous System The MIT Press, Cambridge, Massachusetts, 1992.

[19] von Holst E, Quantitative Untersuchungen über Umstimmungsvorgänge im Zentralnervensystem. I. Der Einfluss des "Appetits" auf das Gleichgewichtsverhalten bei Pterophyllum Zeit Physiol 31: 134-148, 1948.

[20] Magnus G, Keizer J, Minimal Model of -cell $\mathrm{Ca}^{2+}$ Handling Am J Physiol 273 (Cell Physiol 42) : C717-C733, 1997.

[21] Magnus G, Keizer J, Model of -cell Mitochondrial Calcium Handling and Electrical Activity. I. Cytoplasmic Variables Am J Physiol 274 (Cell Physiology 43): C1158C1173, 1998.

[22] Magnus G, Keizer J, Model of -cell Mitochondrial Calcium Handling and Electrical Activity. II. Mitochondrial Variables Am J Physiol 274 (Cell Physiology 43): C1174C1184, 1998.

[23] McCollum G, Navigating a Set of Discrete Regions in Body Position Space $J$ Theor Biol 167: 263-271, 1994.

[24] McCollum G, Mutual Causality and the Generation of Biological Control Systems Int 
$J$ Theor Phys 38: 3253-3267, 1999.

[25] McCollum G, Sensory and Motor Interdependence in Postural Adjustments $J$ Vest Res 9: 303-325, 1999.

[26] McCollum G, Social Barriers to a Theoretical Neuroscience Trends Neurosci 23: 334$336,2000$.

[27] McCollum G, Mathematics Reflecting Sensorimotor Organization. Biol Cybern 88: 108-128, 2003.

[28] McCollum G, Sensorimotor Coordination and the Structure of Space. J Vest Res 13: 157-172, 2003b.

[29] McCollum G, Boyle R, Conditional Transitions in Gaze Dynamics: Role of Vestibular Nuclei in Eye-Only and Eye/Head Gaze Behaviors. Biol Cybern 85: 423-36, 2001.

[30] McCollum G, Boyle R, Rotations in a Vertebrate Setting: Evaluation of the Symmetry Group of the Disynaptic Canal-neck Projection. Biol Cybern 90: 203-217, 2004.

[31] McCollum G, Systems of Logical Systems: Neuroscience and Quantum Logic. Found Sci 7(1): 49-72, 2002.

[32] McCollum G, Holroyd C, Castelfranco AM, Forms of Early Walking J Theor Biol 176: 373-390, 1995.

[33] McCollum G, Shupert CL, Nashner LM, Organizing Sensory Information for Postural Control in Altered Sensory Environments J Theor Biol 180: 257-270, 1996.

[34] McGeer T, Dynamics and Control of Bipedal Locomotion J Theor Biol 163: 277-314, 1993.

[35] McGeer WH Jr, Wall C 3d, Tomko DL, The Chinchilla's Vestibulo-Ocular Reflex Acta Otolaryn 108: 161-167, 1989.

[36] Prochazka A, The Fuzzy Logic of Visuomotor Control Can J Physiol Pharm 74: 456462, 1996.

[37] Prochazka A, Sensorimotor Gain Control: A Basic Strategy of Motor Systems? Prog Neurobiol33: 281-307, 1989.

[38] Prochazka A, Hulliger M, Zangger P, Appentang K, Fusimotor Set: New Evidence for Independent Control of Motoneurones during Movement in the Awake Cat Brain Res 339: 136-140, 1985.

[39] Rinzel J, A Formal Classification of Bursting Mechanisms in Excitable Systems. in Teramoto E, Yamaguti M (eds.) Mathematical Topics in Population Biology, Morphogenesis, and Neurosciences Lecture Notes in Mathematics 71, Springer-Verlag, New York, 1987.

[40] Roberts PD, McCollum G, Dynamics of the Sit-to-Stand Movement Biol Cybern 74: 147-157, 1996.

[41] Roberts PD, McCollum G, The Stomatogastric Nervous System: A Formal Approach Neurosci 72: 1089-1105, 1996.

[42] Schöner G, A Dynamic Theory of Coordination of Discrete Movement Biol Cybern 63: 257-270, 1990.

[43] Schöner G, Haken H, Kelso JAS, A Stochastic Theory of Phase Transitions in Human Hand Movement Biol Cybern 53: 247-257, 1986.

[44] Schöner G, Jiang WY, Kelso JAS, A synergetic theory of quadrupedal gaits and gait transitions. J Theor Biol 142: 359-391, 1990.

[45] Thelen E, Smith LB, A Dynamic Systems Approach to the Development of cognition 
February 25, 2005 13:30 WSPC/INSTRUCTION FILE mccollum04

and Action, The MIT Press, Cambridge, Massachusetts, 1995.

[46] Winfree AT, The Geometry of Biological Time Springer-Verlag, New York, 1980. 